Short Communication

\title{
Evaluation of Mustard Genotypes Under Late Sowing Condition in the Southern Region of Bangladesh
}

\author{
M. S. Uddin ${ }^{1}$, M. M. R. Talukdar, M. S. I. Khan, M. I. Huq, and M. A. Razzaque \\ Regional Agricultural Research Station, Bangladesh Agricultural Research Institute \\ Rahmatpur, Barisal-8211, Bangladesh
}

Received 30 May 2009, accepted in final revised form 27 July 2009

\begin{abstract}
The experiment was conducted to find out suitable mustard genotypes under late sowing condition for the southern region of Bangladesh. Ten genotypes of Rai groups were sown at five different dates (December 01, December 08, December 15, December 22 and December 29). The highest yield was obtained from December 01 sowing whereas the lowest yield was recorded from December 29 sowing. Ishurdi local and Pucca Rai produced higher yield of $924 \mathrm{~kg} / \mathrm{ha}$ and $812 \mathrm{~kg} / \mathrm{ha}$, respectively and these two genotypes could be sown late up to December 15 for obtaining profitable yield.
\end{abstract}

Keywords: Late sowing; Mustard genotypes; Sowing time; Screening.

(c) 2009 JSR Publications. ISSN: 2070-0237 (Print); 2070-0245 (Online). All rights reserved.

DOI: $10.3329 /$ jsr.v1i3.2576

J. Sci. Res. 1 (3), 667-672 (2009)

\section{Introduction}

Oils and fat play important role in human nutrition. As a high energy component of food, edible oil is important for meeting the calorie requirement. This is also important for improving the taste of a number of food items. Fats and oils act as carrier for fat soluble vitamins (A, D, E and K) in the body and therefore, the presence of some fats/oils in the diet is essential for their absorption. In Bangladesh, there are more than ten different oil crops which produce fats and oils of variable quality and quantity. Among them, oleriferous Brassica is an important source of vegetable fats, mainly represented by rapes (Brassica campestris L. and B. napaus L.) and mustard (B. juncea L. Czem and Coss) known as mustard together. Mustard as the main oilseed crop in Bangladesh covers about $60 \%$ of the oilseed area and $61 \%$ of the total production [1].

${ }^{1}$ Corresponding author: mdsaleh03@yahoo.com 
The southern region is characterized by tidal flooding, high rainfall in monsoon and short winter periods. T. aman is the main crop in the cropping pattern. During the rabi season, land remains fallow due to delay harvest of $\mathrm{T}$. aman and excessive soil moisture. Land becomes free and soil comes to working condition at the end of November to first week of January, which is not optimum for sowing of many rabi crops.

Early planting of mustard reduces seed yield as because of excessive growth before flowering due to longer duration of vegetative phases. Plants produce more number of branches bearing excessive pods, resulting in both inter-and intra-pod and seed competition within plants and thus a high rate of seed abortion occurs. On the other hand, plants due to delayed sowing more rapidly fulfill the low temperature needs to initiate earlier inflorescence and flowering. That restricts leaf production ultimately and results in smaller plants and fewer pod bearing branches and finally low yield [2, 3]. Bangladesh Agricultural Research Institute (BARI) has developed high yield potential varieties of rapes and mustard for optimum sowing. Middle of October is the most suitable time for sowing of rapes and mustard in Bangladesh $[4,5]$. B. juncea genotypes, also known as Rai group are usually late potential [6]. Razzaque et al. [7] obtained profitable yield of two varieties of Rai group up to 30 November. The present study deals screening of the genotypes of Rai group under late sowing condition in the southern region of Bangladesh.

\section{Materials and Methods}

The experiment was conducted at Regional Agricultural Research Station, Rahmatpur, Barisal during rabi 2002-03 and 2003-04. The experiment comprised five sowing dates viz. December 01, December 08, December 15, December 22 and December 29 of 2002 and 2003 and ten varieties/lines of Rai group viz., BJ-08, BJ -17, BJ-49, J-535-2, Pucca Rai, Bolder, Jamalpur-1, Ishurdi local, BARI sorisha-9 and Daulat. The treatments were laid out in a split-plot design by assigning the sowing dates in main plots and varieties in sub-plots of $4 \times 1.2 \mathrm{~m}$ with three replications. Recommended doses of fertilizers were applied. Intercultural operations were done as and when necessary. Data on different yield and yield contributing characters were recorded and analyzed statistically.

\section{Results and Discussion}

\section{Effect of sowing time}

The highest yield (1469 kg/ha) was obtained from first sowing (December 01) (Table 1) followed by second sowing (1048 kg/ha), third sowing (607 kg/ha). The fourth and fifth sowing produced the lowest yield. The yield scored $14.5 \%$ coefficient of variation. This revealed there are scopes to screen variety/line for late sowing condition. First sowing produced the tallest plant $(140 \mathrm{~cm})$ and the highest number of siliqua plant (145.5). On the other hand fifth sowing had the shortest $(82 \mathrm{~cm})$ height and the lowest number of siliqua per plant (54). 


\section{Effect of genotypes}

Pucca Rai and Ishurdi local produced the highest yield (Table 2). Bolder, Daulat and J$535-2$ ranked the second position regarding yield. The lowest yield was recorded from BJ17 and BJ-49. Ishurdi local, Jamalpur-1 and Bolder were late in maturity and required 9394 days. BARI sorisha- 10 and Daulat were the earliest in maturity. Pucca Rai and Bolder produced the tallest plant whereas BARI sorisha- 10 produced the shortest $(98 \mathrm{~cm})$. The highest number of siliqua per plant was obtained from Pucca Rai and Ishurdi local. BJ-49 produced the lowest number of siliqua (69.2) per plant.

Table1. Yield and yield components of mustard as affected by sowing dates (pooled average of 2002-03 and 2003-04).

\begin{tabular}{lcccc}
\hline \multicolumn{1}{c}{ Sowing time } & $\begin{array}{c}\text { Yield } \\
(\mathrm{kg} / \mathrm{ha})\end{array}$ & $\begin{array}{c}\text { Days to } \\
\text { maturity }\end{array}$ & $\begin{array}{c}\text { Plant height } \\
(\mathrm{cm})\end{array}$ & No.of siliqua/plant \\
\hline $1^{\text {st }}$ sowing (Dec.01) & $1469 \mathrm{a}$ & 94 & $140 \mathrm{a}$ & $145.5 \mathrm{a}$ \\
$2^{\text {nd }}$ sowing (Dec.08) & $1048 \mathrm{~b}$ & 94 & $135 \mathrm{ab}$ & $131.6 \mathrm{~b}$ \\
$3^{\text {rd }}$ sowing (Dec.15) & $607 \mathrm{c}$ & 92 & $130 \mathrm{~b}$ & $126.2 \mathrm{~b}$ \\
$4^{\text {th }}$ sowing (Dec.22) & $193 \mathrm{~d}$ & 90 & $95 \mathrm{c}$ & $75.3 \mathrm{c}$ \\
$5^{\text {th }}$ sowing (Dec.29) & $183 \mathrm{~d}$ & 86 & $82 \mathrm{~d}$ & $54.0 \mathrm{~d}$ \\
F-test & $* *$ & $\mathrm{~ns}$ & $* *$ & $* *$ \\
CV (\%) & 14.5 & 2.3 & 6.5 & 11.2 \\
\hline
\end{tabular}

Table 2. Yield and yield components of mustard as affected by genotypes (pooled average of 2002-03 and 2003-04).

\begin{tabular}{ccccc}
\hline Genotypes & $\begin{array}{c}\text { Yield } \\
(\mathrm{kg} / \mathrm{ha})\end{array}$ & $\begin{array}{c}\text { Days to } \\
\text { maturity }\end{array}$ & $\begin{array}{c}\text { Plant } \\
\text { height }\end{array}$ & No.of siliqua/plant \\
\hline BJ-08 & $529 \mathrm{~cd}$ & $91 \mathrm{~b}$ & $124 \mathrm{~b}$ & $80.7 \mathrm{~b}$ \\
BJ-17 & $486 \mathrm{~d}$ & $91 \mathrm{~b}$ & $116 \mathrm{c}$ & $93.8 \mathrm{c}$ \\
BJ-49 & $502 \mathrm{~d}$ & $90 \mathrm{~b}$ & $180 \mathrm{~b}$ & $69.2 \mathrm{~d}$ \\
J-535-2 & $754 \mathrm{~b}$ & $91 \mathrm{~b}$ & $115 \mathrm{c}$ & $85.3 \mathrm{~b}$ \\
Pucca Rai & $895 \mathrm{a}$ & $95 \mathrm{a}$ & $138 \mathrm{a}$ & $92.5 \mathrm{a}$ \\
Bolder & $778 \mathrm{~b}$ & $93 \mathrm{a}$ & $135 \mathrm{a}$ & $82.2 \mathrm{~b}$ \\
Jamalpur-1 & $795 \mathrm{~b}$ & $93 \mathrm{a}$ & $100 \mathrm{~d}$ & $88.5 \mathrm{ab}$ \\
Ishurdi local & $897 \mathrm{a}$ & $94 \mathrm{a}$ & $120 \mathrm{~b}$ & $90.8 \mathrm{a}$ \\
BARI sorisha-10 & $621 \mathrm{c}$ & $88 \mathrm{c}$ & $98 \mathrm{~d}$ & $78.5 \mathrm{c}$ \\
Daulat & $745 \mathrm{~b}$ & $89 \mathrm{c}$ & $125 \mathrm{~b}$ & $83.3 \mathrm{~b}$ \\
F-test & $* *$ & $* *$ & & $* *$ \\
\hline
\end{tabular}

\section{Interaction between sowing dates and genotypes}

In first sowing Pucca Rai produced the highest yield (1945 kg/ha) and that was identical with that of Ishurdi local (1736 kg/ha) (Table 3). The lowest yield was recorded from BJ- 
17 (1043 kg/ha). Pucca Rai, Jamalpur-1, Bolder and Ishurdi local were late in maturity (98 days). BJ-08, BJ-17, Bari sorisha-10 and Daulat were the earliest genotypes and required 90 days to maturity. In second sowing (8 December), Ishurdi local gave the highest yield (1529 kg/ha). Jamalpur-1 produced the second highest yield (1388 kg/ha). Days to maturity in second sowing were same with first sowing. In third sowing, Ishurdi local produced the highest yield (924 kg/ha). Pucca Rai was the second highest yielding genotype (812 kg/ha) followed by Jamalpur-1 (742 kg/ha). These genotypes were late in maturity (94 days). In fourth (22 December) and fifth (29 December) sowing, the performances of the genotypes were not satisfactory.

Table 3. Interaction effect of sowing time and genotypes on yield and maturity time of mustard (pooled average of 2002-03 and 2003-04).

\begin{tabular}{|c|c|c|c|c|c|c|c|c|c|c|}
\hline \multirow[t]{2}{*}{ Genotypes } & \multicolumn{2}{|c|}{$\begin{array}{c}\text { First sowing } \\
\left(1^{\text {st }} \text { Dec. }\right)\end{array}$} & \multicolumn{2}{|c|}{$\begin{array}{l}2^{\text {nd }} \text { sowing } \\
\left(8^{\text {th }} \text { Dec. }\right)\end{array}$} & \multicolumn{2}{|c|}{$\begin{array}{l}3^{\text {rd }} \text { sowing } \\
\left(15^{\text {th }} \text { Dec. }\right)\end{array}$} & \multicolumn{2}{|c|}{$\begin{array}{l}4^{\text {th }} \text { sowing } \\
\left(22^{\text {nd }} \text { Dec. }\right)\end{array}$} & \multicolumn{2}{|c|}{$\begin{array}{l}5^{\text {th }} \text { sowing } \\
\left(29^{\text {th }} \text { Dec. }\right)\end{array}$} \\
\hline & $\begin{array}{l}\text { Yield } \\
\text { (kg/ha) }\end{array}$ & $\mathrm{DM}$ & $\begin{array}{l}\text { Yield } \\
\text { (kg/ha) }\end{array}$ & $\mathrm{DM}$ & $\begin{array}{c}\text { Yield } \\
\text { (kg/ha) }\end{array}$ & $\mathrm{DM}$ & $\begin{array}{l}\text { Yield } \\
\text { (kg/ha) }\end{array}$ & $\mathrm{DM}$ & $\begin{array}{c}\text { Yield } \\
\text { (kg/ha) }\end{array}$ & $\mathrm{DM}$ \\
\hline BJ-08 & $1251 \mathrm{~d}$ & 90с & $650 \mathrm{gh}$ & $90 c$ & $374 \mathrm{e}$ & $90 \mathrm{~b}$ & 142 & $87 b$ & 241 & $89 b$ \\
\hline BJ-17 & $1043 \mathrm{f}$ & 91c & 663gh & $90 c$ & $371 \mathrm{e}$ & $90 \mathrm{~b}$ & 179 & $87 b$ & 173 & $88 \mathrm{ab}$ \\
\hline BJ-49 & 1181de & $94 a b$ & $542 \mathrm{i}$ & $94 a b$ & $320 \mathrm{e}$ & $90 \mathrm{~b}$ & 204 & $86 c$ & 264 & $88 \mathrm{ab}$ \\
\hline $\mathrm{J}-535-2$ & 1598bc & $95 \mathrm{ab}$ & 1254cd & $94 a b$ & $634 c$ & $94 a$ & 165 & $88 b$ & 117 & $87 \mathrm{a}$ \\
\hline Pucca Rai & $1944 a$ & $98 \mathrm{a}$ & $1298 c$ & $97 a$ & $812 b$ & $94 a$ & 209 & $94 a$ & 211 & $88 \mathrm{ab}$ \\
\hline Bolder & $1702 \mathrm{~b}$ & $97 \mathrm{a}$ & $1108 d$ & $97 \mathrm{a}$ & $669 c$ & 93a & 265 & $94 a$ & 145 & 87 a \\
\hline Jamalpur-1 & 1529 bc & $98 a$ & $1388 b$ & $97 a$ & $742 \mathrm{bc}$ & $94 a$ & 207 & $94 a$ & 108 & 87 a \\
\hline Ishurdi local & 1736ab & $98 \mathrm{a}$ & 1529a & $97 \mathrm{a}$ & 924 a & $94 a$ & 177 & $95 a$ & 117 & 87 a \\
\hline Barisorisha-10 & $1388 c$ & 90c & 956d-g & 90c & 539cd & $90 \mathrm{~b}$ & 136 & $86 \mathrm{bc}$ & 135 & $88 \mathrm{ab}$ \\
\hline Daulat & $1316 c$ & $90 \mathrm{c}$ & 1097d-g & $90 \mathrm{c}$ & $621 \mathrm{~cd}$ & $90 \mathrm{~b}$ & 249 & $89 b$ & 271 & $88 \mathrm{ab}$ \\
\hline F-test & $* *$ & * & $* *$ & * & $* *$ & $*$ & ns & $*$ & ns & * \\
\hline
\end{tabular}

$\mathrm{DM}=$ Days to maturity.

The present findings may be compared with the findings of several workers [4, 5, 79]. They reported October to November as the best sowing time of mustard and rapeseeds. Razzaque et al. [10] found two genotypes of Rai group, Daulat and Ishurdi local could be late potential and stated that they could be sown up to 30 November for obtaining a profitable yield.

\section{Economics}

The highest value for BCR of Pucca Rai in December 01 sowing was obtained 3.11followed by that of Ishurdi local of the same sowing date (Table 4). Considering the benefit cost ratio, it was observed that most of the genotypes produced economically viable yield up to 08 December sowing. Sowing up to 15 December only Pucca rai and Ishurdi local were able to produce profitable yield with BCR 1.48 and 1.30, respectively. All the genotypes failed to produce viable yield after 15 December sowing. 
Table 4. Economic performance of different mustard genotypes during the rabi seasons of 2002-03 and 2003-04.

\begin{tabular}{|c|c|c|c|c|c|c|c|c|c|}
\hline \multirow[t]{2}{*}{ Sl. } & \multirow[t]{2}{*}{ Genotypes } & \multicolumn{4}{|c|}{ First sowing ( $1^{\text {st }}$ Dec.) } & \multicolumn{4}{|c|}{$2^{\text {nd }}$ sowing ( $8^{\text {th }}$ Dec.) } \\
\hline & & $\begin{array}{l}\text { Yield } \\
\text { (kg/ha) }\end{array}$ & $\begin{array}{c}\text { Gross } \\
\text { return } \\
\text { (Tk/ha) }\end{array}$ & $\begin{array}{c}\text { TVC } \\
\text { (Tk/ha) }\end{array}$ & BCR & $\begin{array}{l}\text { Yield } \\
\text { (kg/ha) }\end{array}$ & $\begin{array}{c}\text { Gross } \\
\text { return } \\
\text { (Tk/ha) }\end{array}$ & $\begin{array}{c}\text { TVC } \\
\text { (Tk/ha) }\end{array}$ & BCR \\
\hline 1. & BJ-08 & 1251 & 31275 & 15629 & 2.00 & 650 & 16250 & 15629 & 1.04 \\
\hline 2. & BJ-17 & 1043 & 26075 & 15629 & 1.67 & 663 & 16575 & 15629 & 1.06 \\
\hline 3. & BJ-49 & 1181 & 29525 & 15629 & 1.89 & 542 & 13550 & 15629 & 0.87 \\
\hline 4. & $J-535-2$ & 1598 & 39950 & 15629 & 2.56 & 1254 & 31250 & 15629 & 2.00 \\
\hline 5. & Pucca Rai & 1944 & 48600 & 15629 & 3.11 & 1298 & 32450 & 15629 & 2.08 \\
\hline 6. & Bolder & 1702 & 42550 & 15629 & 2.72 & 1108 & 27700 & 15629 & 1.77 \\
\hline 7. & Jamalpur-1 & 1529 & 38225 & 15629 & 2.45 & 1388 & 34700 & 15629 & 2.22 \\
\hline 8. & Ishurdi local & 1736 & 43400 & 15629 & 2.79 & 1529 & 38225 & 15629 & 2.45 \\
\hline 9. & Barisorisha-9 & 1388 & 34700 & 15629 & 2.22 & 956 & 23900 & 15629 & 1.53 \\
\hline 10. & Daulat & 1316 & 32900 & 15629 & 2.11 & 1097 & 27425 & 15629 & 1.75 \\
\hline
\end{tabular}

Table 4. (Contd.)

\begin{tabular}{|c|c|c|c|c|c|c|c|c|c|c|c|c|}
\hline \multirow[t]{2}{*}{ Sl. } & \multicolumn{4}{|c|}{$3^{\text {rd }}$ sowing $\left(15^{\text {th }}\right.$ Dec. $)$} & \multicolumn{4}{|c|}{$4^{\text {th }}$ sowing $\left(22^{\text {nd }}\right.$ Dec. $)$} & \multicolumn{4}{|c|}{$5^{\text {th }}$ sowing $\left(29^{\text {th }}\right.$ Dec. $)$} \\
\hline & $\begin{array}{l}\text { Yield } \\
\mathrm{kg} / \mathrm{ha}\end{array}$ & $\begin{array}{c}\text { GS } \\
\text { Tk/ha }\end{array}$ & $\begin{array}{l}\text { TVC } \\
\text { Tk/ha }\end{array}$ & BCR & $\begin{array}{l}\text { Yield } \\
\mathrm{kg} / \mathrm{ha}\end{array}$ & $\begin{array}{c}\text { GS } \\
\text { Tk/ha }\end{array}$ & $\begin{array}{l}\text { TVC } \\
\text { Tk/ha }\end{array}$ & BCR & $\begin{array}{l}\text { Yield } \\
\text { kg/ha }\end{array}$ & $\begin{array}{c}\text { GS } \\
\text { Tk/ha }\end{array}$ & $\begin{array}{l}\text { TVC } \\
\text { Tk/ha }\end{array}$ & BCR \\
\hline 1. & 374 & 9350 & 15629 & 0.60 & 142 & 3550 & 15629 & 0.23 & 241 & 6025 & 15629 & 0.39 \\
\hline 2. & 371 & 9275 & 15629 & $0 . .59$ & 179 & 4475 & 15629 & 0.29 & 173 & 4325 & 15629 & 0.28 \\
\hline 3. & 320 & 8000 & 15629 & $0 . .51$ & 204 & 5100 & 15629 & 0.33 & 264 & 6600 & 15629 & 0.04 \\
\hline 4. & 634 & 15850 & 15629 & 1.01 & 165 & 4125 & 15629 & 0.26 & 117 & 2925 & 15629 & 0.19 \\
\hline 5. & 812 & 20300 & 15629 & 1.30 & 209 & 5225 & 15629 & 0.33 & 211 & 5275 & 15629 & 0.34 \\
\hline 6. & 669 & 16775 & 15629 & 1.02 & 265 & 6625 & 15629 & 0.42 & 145 & 3625 & 15629 & 0.23 \\
\hline 7. & 742 & 18550 & 15629 & 1.17 & 207 & 5175 & 15629 & 0.33 & 108 & 2700 & 15629 & 0.17 \\
\hline 8. & 924 & 23100 & 15629 & 1.48 & 177 & 4425 & 15629 & 0.28 & 117 & 2925 & 15629 & 0.19 \\
\hline 9. & 539 & 13475 & 15629 & 0.86 & 136 & 3400 & 15629 & 0.22 & 135 & 3375 & 15629 & 0.22 \\
\hline 10. & 621 & 15525 & 15629 & $0 . .99$ & 249 & 6225 & 15629 & 0.40 & 271 & 6775 & 15629 & 0.43 \\
\hline
\end{tabular}

TVC $=$ Total variable cost; $\mathrm{BCR}=$ Benefit cost ratio; $\mathrm{GS}=$ Gross return.

Seed rate $=8 \mathrm{~kg} / \mathrm{ha}$

$\begin{array}{lllll}\text { Input } & : & \mathrm{Tk} / \mathrm{kg} & \text { Out put } & : \mathrm{Tk} / \mathrm{kg} \\ \text { Seed } & : & 50.00 & \text { Mustard } & : 30.00 \\ \text { Urea } & : & 7.00 & & \\ \text { TSP } & : & 15.00 & & \\ \text { MP } & : & 10.00 & & \\ \text { Gypsum } & : & 8.00 & \end{array}$

\section{Conclusion}

From the present findings it may be said that Ishurdi local and Pucca Rai of Rai group mustard is suitable for sowing up to 15 December profitably, particularly in the Southern region of Bangladesh.

\section{References}

1. BBS (Bangladesh Bureau of Statistics). In: krishi Dairy 2007 (AIS. DAE, Khamebari, Dhaka, 2006).

2. N. N. Mendham, J. Russel, and N. K. Jarosz, J. Agric. Sci. Camb. 114 (3), 275 (1990). 


\section{Evaluation of Mustard Short Communication}

doi:10.1017/S002185960007266X

3. N. K. Scott, E. A. Ogunremi, J. D. Ivins, and N. J. Mendham, J. Agric. Sci. Camb. 81, 277 (1973). doi:10.1017/S0021859600058937

4. M. A. Hossain, S. Arabinda, and M. J. Abedin, Bangladesh. J. Agric. Res. 9, 54 (1984).

5. M. M. Uddin, A. Samad, M. R. Khan, S. Begum, and M. A. Salam, Bangladesh J. Sci. Inds. Res. 21, 159 (1986).

6. A. Akber, M. Mondol, P. Podder, and H. Ahmed, Booklet (in Bangali) - Oilseed Research Centre. BARI, Joydebpur, Gazipur (1994).

7. M. A. Razzaque, M. A. Howlader, S. Rafiquzzaman, M. Ahsanullah, and M. N. Uddin, Bangladesh J. Agric. Res. 27 (3), 479 (2002).

8. M. J. Mendham and R. K. Scott, J. Agric. Sci. Camb. 84 (1), 487 (1975).

9. B. L. Nag, M. Karim, N. Islam, and M. A. Quddus, Progressive Agriculture. 11 (1\&2), 45 (2000).

10. M. A. Razzaque, M. R. Talukder, S. Uddin, S. I. Khan, and A. Hossain, Bangladesh J. Sci. Inds. Res. 42 (4), 441 (2002). 